\title{
The reversal effect of physical exercise on aging-related increases in APPL2 content in skeletal muscle
}

\author{
Paulo Henrique Canciglieri ${ }^{\mathrm{a}, 1}$, Gabriel Keine Kuga ${ }^{\mathrm{b}, 1}$, Vitor Rosetto Muñoz ${ }^{\mathrm{a}}$, \\ Rafael Calais Gaspar ${ }^{\mathrm{a}}$, Alisson Luiz da Rocha ${ }^{\mathrm{c}}$, Leonardo Breda ${ }^{\mathrm{a}}$, Chadi Pellegrini Anaruma ${ }^{\mathrm{b}}$, \\ Luciele Guerra Minuzzi ${ }^{\mathrm{a}}$, Adelino Sanchez Ramos da Silva ${ }^{\mathrm{c}}$, Dennys Esper Cintra ${ }^{\mathrm{d}, \mathrm{e}}$, \\ Leandro Pereira de Moura ${ }^{\mathrm{a}, \mathrm{b}, \mathrm{e}, \mathrm{f}}$, Eduardo Rochete Ropelle ${ }^{\mathrm{a}, \mathrm{e}, \mathrm{f}}$, José Rodrigo Pauli ${ }^{\mathrm{a}, \mathrm{e}, \mathrm{f}, *}$ \\ ${ }^{a}$ Laboratory of Molecular Biology of Exercise (LaBMEx), School of Applied Sciences, University of Campinas (UNICAMP), Limeira, SP, Brazil \\ b Post-graduate Program in Movement Sciences, São Paulo State University (UNESP), Rio Claro, SP, Brazil \\ ${ }^{\mathrm{c}}$ School of Physical Education and Sport of Ribeirao Preto, University of Sao Paulo, Ribeirao Preto, SP, Brazil \\ ${ }^{\mathrm{d}}$ Laboratory of Nutritional Genomics (LabGeN), School of Applied Sciences, University of Campinas (UNICAMP), Limeira, SP, Brazil \\ ${ }^{\mathrm{e}}$ Laboratory of Cell Signaling, Obesity and Comorbidities Research Center (OCRC), University of Campinas, Campinas, SP, Brazil \\ ${ }^{\mathrm{f}}$ CEPECE - Center of Research in Sport Sciences, School of Applied Sciences, University of Campinas (UNICAMP), Limeira, SP, Brazil
}

\section{A R T I C L E I N F O}

\section{Keywords:}

Aging

APPL1

APPL2

Insulin

Skeletal muscle

Physical exercise

\begin{abstract}
A B S T R A C T
Aims: The aim of this study was to evaluate the effects of aging on intracellular adiponectin signaling and the possible therapeutic effect of physical exercise.

Main methods: Fischer 344 rats were distributed in the following groups: Young (3 months old); Sedentary Old (Old, 27 months old); and Old Exercised (Old-Exe, 27 months old), which were subjected to a short-term exercise training protocol.

Key findings: The results showed that the old rats presented glucose intolerance without increased adiposity. However, short-term exercise training reversed this disorder, which was associated with a decrease in the pleckstrin homology domain, phosphotyrosine-binding domain, and leucine zipper motif (APPL) isoform 2 (APPL2) content. The APPL isoform 1 (APPL1) and TRB3 (Tribbles homolog 3) contents were not altered. Akt phosphorylation was only increased in the old exercised rats. There was a reduction in the content of adiponectin receptor 1 in the old rats.

Significance: The short-term exercise training protocol was able to decrease APPL2 content in the skeletal muscle, which was accompanied by an improvement in the glucose tolerance of the old Fischer 344 rats. These findings provide new evidence supporting the role of physical exercise as a non-pharmacological therapeutic intervention to attenuate age-related deficits.
\end{abstract}

\section{Introduction}

Aging is a natural and unmodifiable process that has been related to low grade inflammation and insulin resistance in skeletal muscle, a condition called inflammaging [1-3]. This process is one of the main causes of Type 2 Diabetes Mellitus due to the reduction in glucose uptake in skeletal muscle, inducing chronic hyperglycemia and favoring the development of associated comorbidities [1-3]. Therefore, the scientific community should elucidate the molecular mechanisms that are responsible for glucose uptake as well as the agents involved in the breakdown of this process.

A well-known amplifier of insulin signaling is adiponectin [4,5]. In fact, adiponectin activates adiponectin receptors 1 and 2 (AdipoR 1 and AdipoR2) [5,6], recruiting the isoform 1 content of the adaptor protein containing the pleckstrin homology domain, phosphotyrosine-binding domain, and leucine zipper motif (APPL1) protein that is able to compete with Tribbles homolog 3 (TRB3) for interaction with Protein Kinase B (Akt) [7]. TRB3 is a pseudo-kinase that, when associated with Protein Kinase B (Akt) [7], inhibits its phosphorylation and the consequent insulin-mediated glucose uptake through Glucose Transporter

* Corresponding author at: School of Applied Sciences, University of Campinas (UNICAMP), R. Pedro Zaccaria, 1300 Caixa Postal 1068, Jd. Sta. Luzia, CEP 13484350 Limeira, Sao Paulo, Brazil.

E-mail address: jose.pauli@fca.unicamp.br (J.R. Pauli).

${ }^{1}$ These authors contributed equally to this work. 
Type 4 (GLUT4) translocation to the plasma membrane [8].

A counterregulatory mechanism of this signaling pathway is through APPL isoform 2 (APPL2) [9]. This protein is able to inhibit the interaction between APPL1 and AdipoRs, turning off adiponectin signaling [9]. In addition, APPL2 has the ability to inhibit TBC1 domain family member 1 (TBC1D1) phosphorylation by Akt [9]. TBC1D1 is a protein of the Rab-GTPase-activating proteins (GAPs) Tre-2/Bub2/ Cdc16 domain family that increases GLUT4 translocation to the plasma membrane, increasing glucose uptake [9].

One of the main therapeutic interventions to restore insulin signaling and increase glucose uptake in skeletal muscle is physical exercise [10]. The protective effects of physical exercise to reduce the activation of inflammatory markers such as c-Jun N-terminal Kinase (JNK) and Inhibitor of Kappa B Kinase (IKK) in elderly animals are well known [10]. Additionally, exercise promotes a positive effect on APPL1 signaling [11-13], suggesting a possible adjacent mechanism in which physical exercise can amplify the glucose uptake in skeletal muscle.

Nevertheless, there is a lack of evidence about the aging-related effects on APPL1 and APPL2 signaling pathways in the skeletal muscle. Therefore, we hypothesized that aging is related to an increase in APPL2 content and a reduction in APPL1 content in the skeletal muscle, increasing the inhibitory association between TRB3 and Akt. Moreover, this descriptive study aimed to evaluate the possible protective effects of a short-term exercise training protocol in attenuating these molecular derangements.

\section{Materials and methods}

\subsection{Experimental animals}

Young ( 3 months old) and old (27 months old) Fischer 344 rats were utilized for the experimental procedures. The rats were kept in collective cages with free access to water and a standard chow diet, at a constant temperature of $21 \pm 2{ }^{\circ} \mathrm{C}$. The old animals were divided into sedentary (Old) and exercised (Old-Exe) groups. These rats were obtained from the Multidisciplinary Center for Biological Investigation in Laboratory Animal Science (CEMIB) at the University of Campinas (UNICAMP). All procedures were approved by the Animal Ethics Committee (CEUA) of the Institute of Biological Sciences at UNICAMP, in Campinas, SP (case number 4531-1/2017).

\subsection{Short-term exercise training protocol}

Initially, the animals underwent a 5-day adaptation period to physical exercise. On days 1 and 2 of adaptation, they remained on the treadmill at a speed of $3 \mathrm{~m} / \mathrm{min}$ for $10 \mathrm{~min}$ with a $10^{\circ}$ inclination. On days 3,4 , and 5 the speed was increased to $6 \mathrm{~m} / \mathrm{min}$, with $10 \mathrm{~min}$ of duration and $10^{\circ}$ inclination. After this, the animals performed the short-term exercise training protocol. The treadmill was programmed to maintain a constant speed of $12 \mathrm{~m} / \mathrm{min}$ with a $10^{\circ}$ inclination. On the first day of exercise, the animals exercised for $15 \mathrm{~min}$, on the second day they exercised for $30 \mathrm{~min}$, and on the third day they exercised for $45 \mathrm{~min}$ (with $10 \mathrm{~min}$ of rest after the initial $30 \mathrm{~min}$ ). On the fourth, fifth, and sixth days, the animals ran for $60 \mathrm{~min}$ with a $10 \mathrm{~min}$ rest after the first $30 \mathrm{~min} .16 \mathrm{~h}$ after the last of these exercise sessions, the animals were submitted to the Glucose Tolerance Test (GTT). The next day, the final exercise session was performed, followed by tissue extraction. The experimental diagram is illustrated in Fig. 1.

\subsection{Fasting glucose and glucose tolerance test (GTT)}

The GTT was performed $16 \mathrm{~h}$ after the previous exercise session, after a fasting period of $8 \mathrm{~h}$. Then, blood samples were collected from the tail (Fasting Glucose). The animals received an intraperitoneal injection of $2 \mathrm{~g} / \mathrm{Kg}$ glucose (Sigma-Aldrich ${ }^{\circledR}$ ) solution and blood samples were collected after 30,60 , and $120 \mathrm{~min}$ for glucose measurement (glucometer Accu-Chek; Roche Diagnostics ${ }^{\circledR}$, Corp. ${ }^{\circledR}$, Indianapolis, IN).

\subsection{Tissue collection, western blotting, and immunoprecipitation analysis}

Anesthesia using ketamine $(100 \mathrm{mg} / \mathrm{kg})$ and xylazine $(5 \mathrm{mg} / \mathrm{kg})$ was performed $16 \mathrm{~h}$ after the last physical exercise session. Then, insulin was administered through the hepatic portal vein. After $90 \mathrm{~s}$, the gastrocnemius was removed and euthanasia was performed. The tissue homogenization and the SDS-PAGE polyacrylamide gel electrophoresis (western blotting) were performed as recently described by our research group $[14,15]$. The nitrocellulose membranes were incubated overnight $\left(4{ }^{\circ} \mathrm{C}\right)$ with the following specific antibodies: Santa Cruz Biotechnology $=$ TRB3 (mouse, sc-390,242); Cell Signaling Technology $=\mathrm{GAPDH}$ (rabbit, \#2118), $\mathrm{pAkt}^{\mathrm{S} 473}$ (rabbit, \#4060), Akt (rabbit, \#4685), APPL1 (rabbit, \#3858); Biorbyt = AdipoR2 (rabbit, orb10047); Novus Biologicals = APPL2 (rabbit, NBP2-16165), and AdipoR1 (rabbit, NBP2-15300). For the immunoprecipitation, the muscle lysate was incubated overnight with anti-Akt (Santa Cruz Biotechnology, sc-8312) antibody associated with protein G-sepharose, and the following procedures were performed as recently described by our research group [15]. The bands were visualized with enhanced chemiluminescence reagent (ECL) and quantified with the UN-SCAN-IT gel $6.1^{\circledast}$ software.

\subsection{Statistical analysis}

For all data, the Gaussian's distribution was assessed using a Kolmogorov-Smirnov test. After this, we performed a Anova one-way (body weight, intraperitoneal fat weight, fasting glucose, area under the curve for GTT, and western blot data) or two way (in GTT test (Groups $\times$ Time)). When different, the Bonferroni post-hoc test was adopted to compare all groups vs Old group, with $5 \%(p<0.05)$ of significance level. The GraphPad Prism 6.0 ${ }^{\circledR}$ software was used to build the graphs and for the statistical analysis.

\section{Results}

\subsection{Physiological and metabolic parameters}

The Fischer rats did not present changes in body weight ( $p=0.7745$ ) (Fig. $2 \mathrm{~A}$ ) and adiposity (Epididymal: $p=0.3801$; Retroperitoneal: $p=0.6140$; Mesenteric: $p=0.7167$; Fig. $2 \mathrm{~B}$ ) with aging. Although the old rats did not present increased fasting glucose (Young vs. Old: $p>0.9999$ ), the animals subjected to the short-term physical exercise protocol had a reduction in this parameter (Old vs. Old-Exe: $p=0.0172$ ) (Fig. 2C). During the GTT, there were significant differences after $30 \mathrm{~min}$ (Young vs. Old, $p=0.0032$; Old vs. Old-Exe, $p<0.0001$ ) and $60 \mathrm{~min}$ (Young vs. Old, $p=0.0009$; Old vs. Old-Exe, $p=0.0071$ ). After the GTT, the old rats presented glucose intolerance and physical exercise was thus efficient in restoring glucose uptake (Young vs. Old, $p=0.0301$; Old vs. Old-Exe, $p=0.0106$ ) (Fig. $2 \mathrm{D}$ and E).

3.2. Short-term exercise training improves Akt phosphorylation and reduces APPL2 content, without changes in APPL1 and TRB3 contents in the skeletal muscle

In our molecular analyses, we verified that the Old-Exe rats presented higher Akt phosphorylation in the skeletal muscle (Fig. 3A and B) than the Old rats (Young vs. Old: $p=0.9909$; Old vs. Old-Exe: $p=0.0340$ ), indicating the positive effects of physical exercise. We did not find any difference in the APPL1 contents among the experimental groups ( $p=0.0669$ ) (Fig. 3A and C). However, aging was associated with increased APPL2 content (Fig. 3A and D) and exercise was efficient in restoring this parameter (Young vs. Old: $p=0.0371$; Old vs. Old-Exe: $p=0.0268$ ). The Old animals had lower AdipoR1 content (Fig. 3A and 
A.

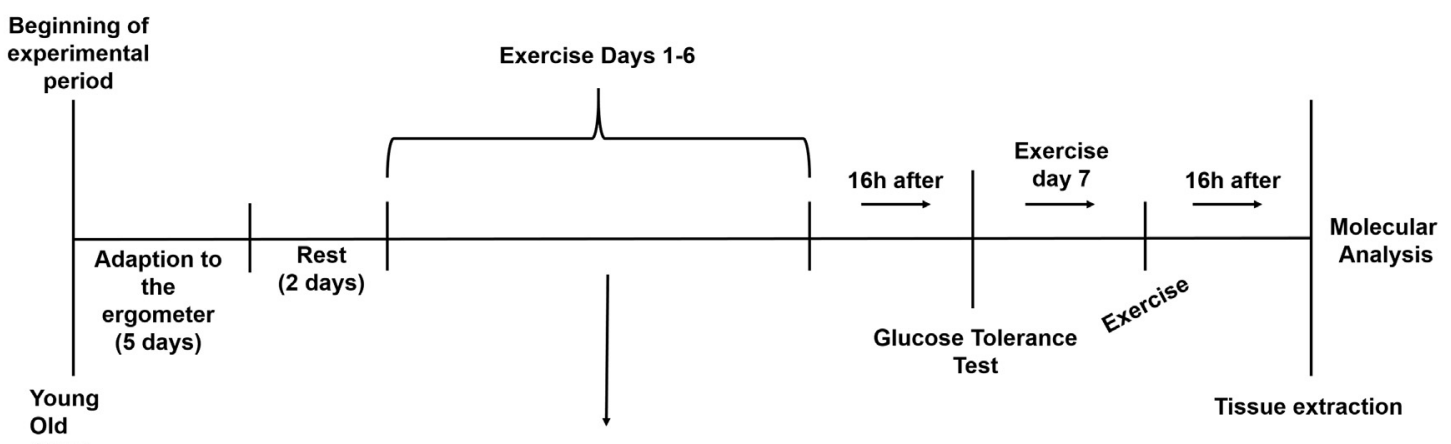

Old-Exe

\section{B. Short-term Exercise Training Protocol}

Treadmill $\left(12 \mathrm{~m} / \mathrm{min}-10^{\circ}\right.$ inclination)

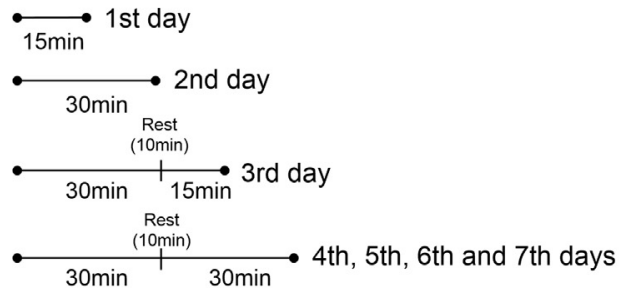

Fig. 1. Illustration of the experimental period (A) and the short-term exercise training protocol (B).

E) and there was no effect of physical exercise on this parameter (Young vs. Old: $p=0.0056$; Old vs. Old-Exe: $p=0.0170$ ). All experimental groups had similar AdipoR2 contents $(p=0.8055)$ (Fig. 3A and F). Finally, we found no difference in the TRB3 $(p=0.8134)$ (Fig. $3 \mathrm{~A}$ and G) content between the groups.

\subsection{Aging does not change the association of TRB3 with Akt in the} gastrocnemius muscle of Fischer rats

To evaluate the association between TRB3 and Akt, we performed the immunoprecipitation assay. However, we did not observe significant changes between the experimental groups for this parameter $(p=0.2471)$ (Fig. 4A and B).

\section{Discussion}

Aging is a biological process strictly associated with a low-grade inflammatory state [1-3]. In our study, we verified that 27 month old Fischer 344 rats did not present significant alterations in body weight and adiposity compared to rats with 3 months of age. Although the Old rats did not present alterations in fasting glucose, they developed glucose intolerance. Physical exercise is one of the main therapeutic
A.

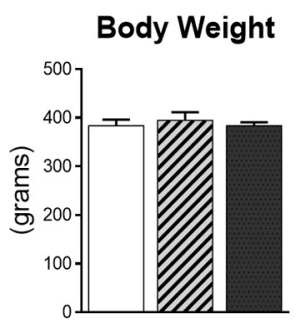

B.

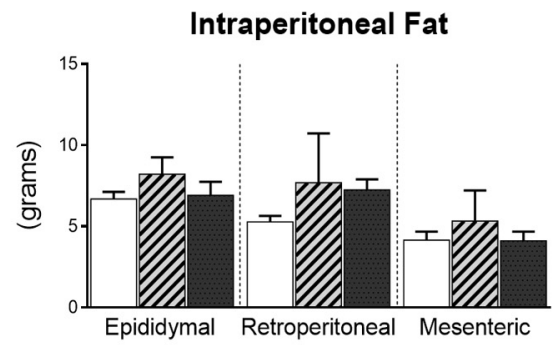

C. Fasting Glucose

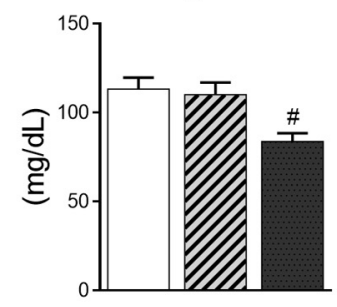

D.

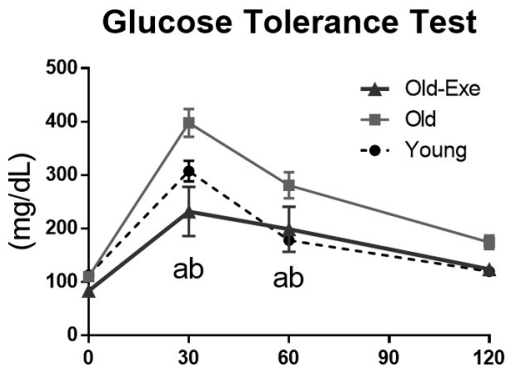

E.

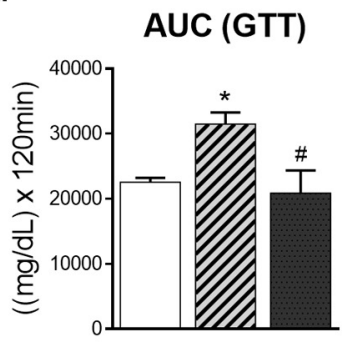

Fig. 2. Physiological parameters. Body weight (A) and adiposity (B); Fasting glucose (C); Glucose tolerance test (GTT) curve (D); Area under the curve for the GTT (E) of the experimental groups. *, $p<0.05$ vs. Young; \#, $p<0.05$ vs. Old; a, $p<0.05$ Young vs. Old; $\mathrm{b}, p<0.05$ Old vs. Old-Exe. $\mathrm{n}=6$ animals per group. 
A.

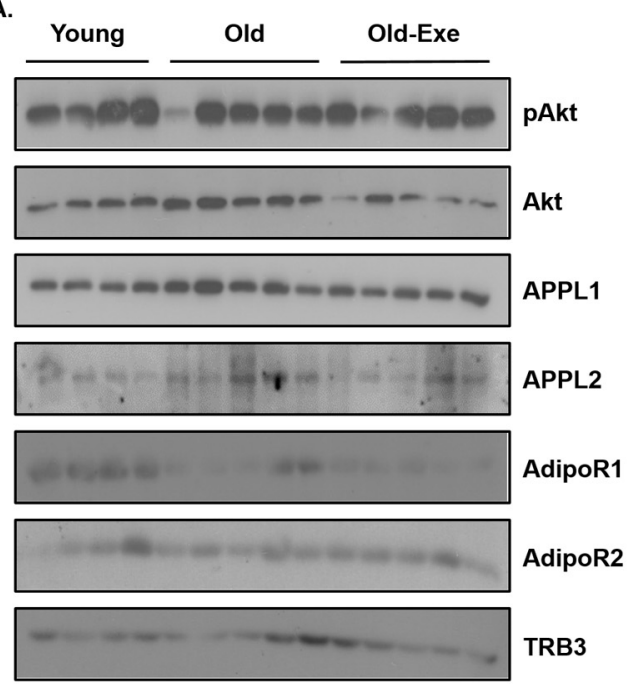

B.

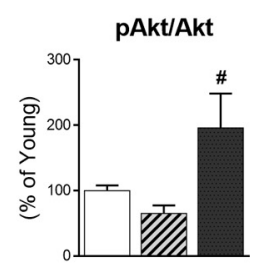

E.

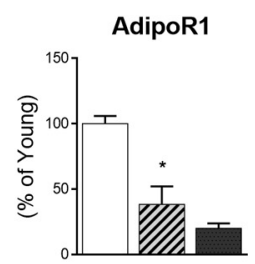

c.

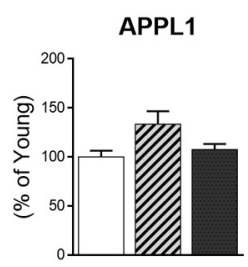

F.

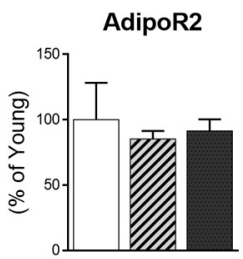

D.

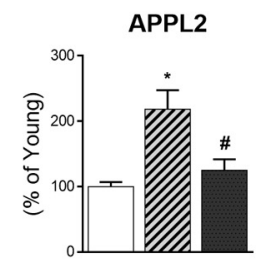

G.

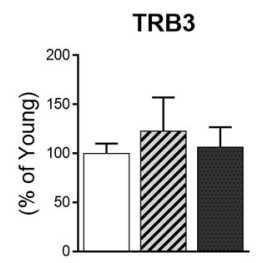

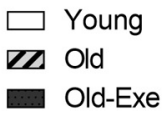

${ }^{*} p<0.05$ vs Young

$\# p<0.05$ vs Old

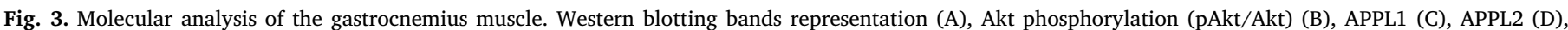

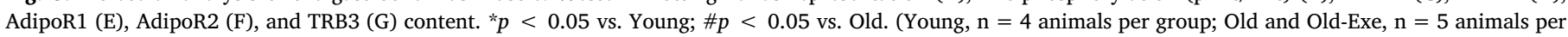
group).

A.

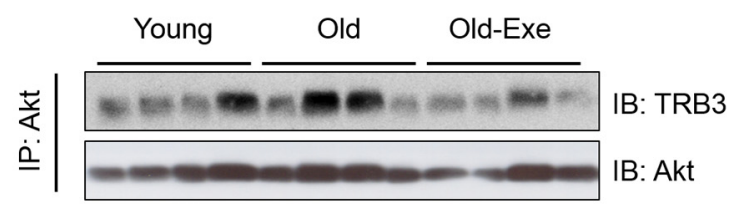

B.

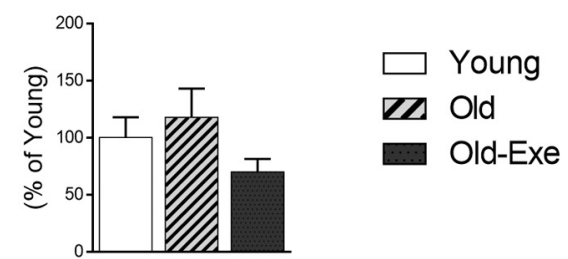

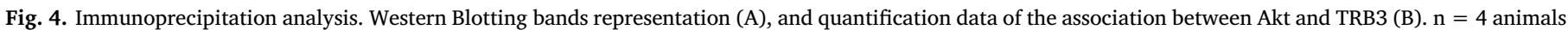
per group.

interventions used to attenuate the decline of biological functions associated with aging $[10,16,17]$. We found that the old rats subjected to the short-term exercise training protocol had reduced fasting blood glucose and increased glucose tolerance without reductions in adiposity or body weight. These improvements in response to physical exercise are associated with increased Akt phosphorylation in the gastrocnemius muscle.

Adiponectin is able to amplify insulin sensitivity through APPL1 interaction with Insulin Receptor Substrate 1/2 [4]. However, there is little evidence regarding the deleterious effects of aging on adiponectin intracellular signaling through APPL1 in the gastrocnemius muscle. In the present study, there was no difference in APPL1 content between the experimental groups. Our findings corroborate a previous study showing no effects of aging on Akt phosphorylation in the serine residue ${ }^{473}$ and APPL1 content in the soleus and epitrochlearis muscles [18]. Therefore, until now, our study is the first to evaluate the effects of aging on the APPL1 content in the gastrocnemius muscle.

In fact, APPL1 is able to prevent the inhibitory interaction of TRB3 with Akt [7]. In contrast with our initial hypothesis, we did not verify any effects of aging or physical exercise on TRB3 content. Nevertheless, we evaluated the association between TRB3 and Akt through the immunoprecipitation assay. Fischer 344 rats apparently do not display significant changes in this mechanism in response to aging or physical exercise. This lack of alteration probably occurred because the experimental animals did not present increased body weight or adiposity with aging, considering that the main trigger for increased TRB3 is the endoplasmic reticulum stress phenomenon [19], a condition strictly associated with obesity [20].

Regarding the effects of aging on adiponectin signaling, this was the first study to show the effects of aging on the APPL2 content in skeletal muscle. As mentioned in our initial hypothesis, we verified that the 27 month old Fischer 344 rats had increased APPL2 content in the gastrocnemius muscle, which was attenuated by physical exercise. APPL2 is able to prevent the phosphorylation of TBC1D1 by Akt, reducing the translocation of GLUT4 to the plasma membrane and the consequent glucose uptake [9]. The reduction of APPL2 content in skeletal muscle may be a new mechanism by which physical exercise can improve glucose tolerance during the aging process.

Also, we evaluated the content of adiponectin receptors 1 and 2 in the gastrocnemius. We showed that aging was associated with AdipoR1 reduction and short-term physical exercise was not efficient in attenuating this derangement. There were no changes in the AdipoR2 content. The fact that only AdipoR1 underwent changes with aging may be a consequence of AdipoR1 being the most important adiponectin receptor in skeletal muscle [6]. Further research is necessary to assess whether long-term physical training is able to restore the AdipoR1 content in the gastrocnemius muscle of aged animals.

In summary, the findings of the present study suggest that Fischer 344 rats become glucose intolerant with aging even without changes in body weight and adiposity. The main protein of the adiponectin 
pathway involved in this physiological derangement is APPL2. Likewise, previous studies have found negative effects of aging on glucose homeostasis and phosphorylation of Akt in important insulin action sites (such as the gastrocnemius and liver) in animal models $[10,21]$. Interestingly, physical exercise was effective in increasing glucose homeostasis and insulin signaling, in addition to reducing inflammatory signaling in these tissues [10,21]. Finally, physical exercise is an interesting therapeutic intervention to attenuate the aging-associated decline of glucose intolerance, and the reduction of the APPL2 content in the gastrocnemius muscle contributes to this process in Fischer 344 rats.

\section{Acknowledgements}

The authors thank to FAPESP (2016/18488-8), and to CNPq (306535/2017-3) to all support during the experiment.

\section{Author contributions}

P.H.C., V.R.M., G.K.K., R.C.G., and L.B. performed the experimental procedures and the manuscript writing. V.R.M., G.K.K., R.C.G., A.L.R, and L.G.M. performed the molecular analyzes. V.R.M., G.K.K. and C.P.A. analyzed the data and built the graphs. A.S.R.S., D.E.C., L.P.M., and E.R.R. reviewed the manuscript. J.R.P. were responsible for the research funding, the laboratory support and final review of the manuscript.

\section{Conflict of interest statement}

The authors declare that there are no conflicts of interest.

\section{References}

[1] C. Franceschi, M. Bonafè, S. Valensin, F. Olivieri, M. De Luca, E. Ottaviani, G. De Benedictis, Inflamm-aging. An evolutionary perspective on immunosenescence, Ann. N. Y. Acad. Sci. 908 (2000) 244-254.

[2] E. Cevenini, D. Monti, C. Franceschi, Inflamm-ageing, Curr. Opin. Clin. Nutr. Metab. Care 16 (2013) 14-20, https://doi.org/10.1097/MCO.0b013e32835ada13.

[3] D. Frasca, B.B. Blomberg, R. Paganelli, Aging, obesity, and inflammatory age-related diseases, Front. Immunol. 8 (2017) 1745, , https://doi.org/10.3389/fimmu. 2017.01745.

[4] J. Ryu, A.K. Galan, X. Xin, F. Dong, M.A. Abdul-Ghani, L. Zhou, C. Wang, C. Li, B.M. Holmes, L.B. Sloane, S.N. Austad, S. Guo, N. Musi, R.A. Defronzo, C. Deng, M.F. White, F. Liu, L.Q. Dong, APPL1 potentiates insulin sensitivity by facilitating the binding of IRS1/2 to the insulin receptor, Cell Rep. 7 (2014) 1227-1238, https://doi.org/10.1016/j.celrep.2014.04.006.

[5] S.S. Deepa, L.Q. Dong, APPL1: role in adiponectin signaling and beyond, Am. J. Physiol. Endocrinol. Metab. 296 (2009) E22-E36, https://doi.org/10.1152/ ajpendo.90731.2008.

[6] T. Yamauchi, J. Kamon, Y. Ito, A. Tsuchida, T. Yokomizo, S. Kita, T. Sugiyama, M. Miyagishi, K. Hara, M. Tsunoda, K. Murakami, T. Ohteki, S. Uchida, S. Takekawa, H. Waki, N.H. Tsuno, Y. Shibata, Y. Terauchi, P. Froguel, K. Tobe, S. Koyasu, K. Taira, T. Kitamura, T. Shimizu, R. Nagai, T. Kadowaki, Cloning of adiponectin receptors that mediate antidiabetic metabolic effects, Nature 423
(2003) 762-769, https://doi.org/10.1038/nature01705.

[7] K.K.Y. Cheng, M.A. Iglesias, K.S.L. Lam, Y. Wang, G. Sweeney, W. Zhu, P.M. Vanhoutte, E.W. Kraegen, A. Xu, APPL1 potentiates insulin-mediated inhibition of hepatic glucose production and alleviates diabetes via Akt activation in mice, Cell Metab. 9 (2009) 417-427, https://doi.org/10.1016/j.cmet.2009.03.013.

[8] A. Matos, E.R. Ropelle, J.R. Pauli, M.J.S. Frederico, R.A. De Pinho, L.A. Velloso, C.T. De Souza, Acute exercise reverses TRB3 expression in the skeletal muscle and ameliorates whole body insulin sensitivity in diabetic mice, Acta Physiol. 198 (2010) 61-69, https://doi.org/10.1111/j.1748-1716.2009.02031.x.

[9] K.K.Y. Cheng, W. Zhu, B. Chen, Y. Wang, D. Wu, G. Sweeney, B. Wang, K.S.L. Lam, A. Xu, The adaptor protein APPL2 inhibits insulin-stimulated glucose uptake by interacting with TBC1D1 in skeletal muscle, Diabetes 63 (2014) 3748-3758, https://doi.org/10.2337/db14-0337.

[10] J.R. Pauli, E.R. Ropelle, D.E. Cintra, C.T. De Souza, A.S.R. da Silva, J.C. Moraes, P.O. Prada, J.A.C. de Almeida Leme, E. Luciano, L.A. Velloso, J.B.C. Carvalheira, M.J.A. Saad, Acute exercise reverses aged-induced impairments in insulin signaling in rodent skeletal muscle, Mech. Ageing Dev. 131 (2010) 323-329, https://doi.org/ 10.1016/j.mad.2010.03.004.

[11] J. Farias, R. Maggi, C. Tromm, L. Silva, T. Luciano, S. Marques, F. Lira, C. de Souza, R. Pinho, Exercise training performed simultaneously to a high-fat diet reduces the degree of insulin resistance and improves adipoR1-2/APPL1 protein levels in mice, Lipids Health Dis. 11 (2012) 134, https://doi.org/10.1186/1476-511x-11-134.

[12] R. Marinho, E.R. Ropelle, D.E. Cintra, C.T. De Souza, A.S.R. Da Silva, F.C. Bertoli, E. Colantonio, V. D'Almeida, J.R. Pauli, Endurance exercise training increases APPL1 expression and improves insulin signaling in the hepatic tissue of diet-induced obese mice, independently of weight loss, J. Cell. Physiol. 227 (2012) 2917-2926, https://doi.org/10.1002/jcp.23037.

[13] R.C. Gaspar, V.R. Muñoz, G.P. Formigari, G.K. Kuga, S.C.B.R. Nakandakari, J.D. Botezelli, A.S.R. da Silva, D.E. Cintra, L.P. de Moura, E.R. Ropelle, J.R. Pauli, Acute physical exercise increases the adaptor protein APPL1 in the hypothalamus of obese mice, Cytokine 110 (2018) 87-93, https://doi.org/10.1016/j.cyto.2018.04. 013.

[14] J.D. Botezelli, A. Coope, A.C. Ghezzi, L.T. Cambri, L.P. Moura, P.P.M. Scariot, R.S. Gaspar, R.A. Mekary, E.R. Ropelle, J.R. Pauli, Strength training prevents hyperinsulinemia, insulin resistance, and inflammation independent of weight loss in fructose-fed animals, Sci. Rep. 6 (2016) 31106, , https://doi.org/10.1038/ srep31106.

[15] V.R. Muñoz, R.C. Gaspar, G.K. Kuga, A.L. da Rocha, B.M. Crisol, J.D. Botezelli, I.L. Baptista, R.A. Mekary, A.S.R. da Silva, D.E. Cintra, L.P. de Moura, E.R. Ropelle, J.R. Pauli, Exercise increases rho-kinase activity and insulin signaling in skeletal muscle, J. Cell. Physiol. (2018), https://doi.org/10.1002/jcp.26278.

[16] A. Rebelo-Marques, A. De Sousa Lages, R. Andrade, C.F. Ribeiro, A. Mota-Pinto, F. Carrilho, J. Espregueira-Mendes, Aging hallmarks: the benefits of physical exercise, Front. Endocrinol. 9 (2018) 258, , https://doi.org/10.3389/fendo.2018. 00258 .

[17] G.D. Cartee, R.T. Hepple, M.M. Bamman, J.R. Zierath, Exercise promotes healthy aging of skeletal muscle, Cell Metab. 23 (2016) 1034-1047, https://doi.org/10. 1016/j.cmet.2016.05.007.

[18] N. Sharma, E.B. Arias, M.P. Sajan, J.G. MacKrell, A.D. Bhat, R.V. Farese, G.D. Cartee, Insulin resistance for glucose uptake and Akt2 phosphorylation in the soleus, but not epitrochlearis, muscles of old vs. adult rats, J. Appl. Physiol. 108 (2010) 1631-1640, https://doi.org/10.1152/japplphysiol.01412.2009.

[19] N. Pierre, R. Fernández-Verdejo, P. Regnier, S. Vanmechelen, B. Demeulder, M. Francaux, IRE1 $\alpha$ and TRB3 do not contribute to the disruption of proximal insulin signaling caused by palmitate in C2C12 myotubes, Cell Biol. Int. 40 (2016) 91-99, https://doi.org/10.1002/cbin.10542.

[20] M. Cnop, F. Foufelle, L.A. Velloso, Endoplasmic reticulum stress, obesity and diabetes, Trends Mol. Med. 18 (2012) 59-68, https://doi.org/10.1016/j.molmed. 2011.07.010.

[21] L.P. de Moura, L.S. Souza Pauli, D.E. Cintra, C.T. de Souza, A.S.R. da Silva, R. Marinho, M.A.R. de Melo, E.R. Ropelle, J.R. Pauli, Acute exercise decreases PTP1B protein level and improves insulin signaling in the liver of old rats, Immun. Ageing 10 (2013) 8, https://doi.org/10.1186/1742-4933-10-8. 